\title{
COVID-19 Treatment Experiences in the ICU
}

\author{
Harish SIddaiah, MD; Shilpadevi S. Patil, MD'; George Mychaskiw II, DO, FAAP, FACOP, FASA ${ }^{1}$ \\ ' Louisiana State University, Shreveport, LA
}

KEYWORDS:
Anesthesiology
Coronavirus
COVID-19
Critical Care
Pandemic

\section{INTRODUCTION}

The COVID-19 pandemic has been a global crisis at an unprecedented level. More than 4.75 million cases and 157,000 deaths have been reported in the U.S. as of August 3, 2020.1,2 The disease process, from symptoms and diagnosis to medications and treatment, has been a challenge as COVID-19 is a novel disease that the world has never before encountered. In this article, the authors discuss the disease symptoms, pathophysiology and treatments from their experience treating COVID positive patients in intensive care units of a major Louisiana academic medical center. Much of this clinical practice has been based on limited evidence from other centers, underpowered clinical trials and empiric clinical judgment from experience with similar pathophysiologic conditions. Over time, we expect the quantity and validation of quality evidence to increase, thus better guide our practice.

Worldwide data indicates individuals of all ages are at risk for infection and severe disease. However, the probability of fatal disease from multiorgan failure secondary to cytokine storm is highest in people aged $\geq 65$ years and those living in a nursing home or long-term care facility. Other individuals at the highest risk for COVID-19 are people of any age with certain underlying conditions, especially when not well-controlled, including:

- Cardiovascular disease

- Hypertension

- Diabetes

\section{CORRESPONDENCE:}

George Mychaskiw II, DO, FAAP, FACOP, FASA I

gmycha@lsuhsc.edu

Copyright $\odot 2020$ by the American College of Osteopathic Family Physicians. All rights reserved. Print ISSN: 1877-573X

DOI: $10.33181 / 12055$
- Chronic respiratory disease

- Cancer

- Renal disease

- Obesity $^{3-7}$

National Institutes of Health $(\mathrm{NIH})$ data estimates that the incubation period for COVID-19 is up to 14 days from the time of exposure, with a median incubation period of four to five days. ${ }^{8,9}$ The spectrum of illness can range from asymptomatic infection to severe pneumonia with acute respiratory distress syndrome (ARDS) and death. In a summary of 72,314 persons with COVID-19 in China, $81 \%$ of cases were reported to be mild, $14 \%$ were severe and $5 \%$ were critical. ${ }^{10}$ In a report of 1,482 hospitalized patients with confirmed COVID-19 in the U.S., the most common presenting symptoms were cough (86\%), fever or chills $(85 \%)$, shortness of breath (80\%), diarrhea (27\%) and nausea (24\%). ${ }^{7}$ Other reported symptoms have included, but are not limited to, sputum production, headache, dizziness, rhinorrhea, anosmia, dysgeusia, sore throat, abdominal pain, anorexia and vomiting.

Common laboratory findings of COVID-19 include leukopenia and lymphopenia. Other laboratory abnormalities have included elevations in aminotransferase levels, C-reactive protein, D-dimer, ferritin and lactate dehydrogenase.

Studies report that acute myocardial injury (7.2-17\%) and acute renal injury (2.9-15\%) can occur in severe patients. The reported incidence of acute respiratory distress syndrome (ARDS) is 15.6$31 \%$, which is notably higher than that of other organ injuries. ${ }^{8-11}$ Additionally, the incidence of coagulation disorder is around 5-10\%.

The most common respiratory symptom of COVID-19 is a dry cough (59.4-82\%). ${ }^{8-11}$ Sputum production is less. It suggests that injury to the alveolar epithelial cells is the main cause of COVID-19-related 
ARDS and endothelial cells are less damaged, with, therefore, less exudation. ${ }^{12}$

Chest imaging findings suggest the involvement of both lungs. Abnormalities in chest $x$-rays vary but typically reveal bilateral multifocal opacities. Abnormalities seen in computed tomography (CT) of the chest also vary but usually reveal bilateral peripheral groundglass opacities, with the development of areas of consolidation later in the clinical course. ${ }^{11}$ Imaging may be normal early in infection but can be abnormal even in the absence of symptoms. ${ }^{11}$

At our institution, COVID-19 positive patients are admitted if they require supplemental oxygen to maintain oxygen saturation above $92 \%$ and also have an increased work of breathing (respiratory distress, increased respiratory rate).

Earlier during the pandemic, all patients with oxygen saturations less than $90 \%$ on supplemental oxygen were intubated. However, we learned from the clinical experience of other institutions worldwide that intubating every hypoxic patient was doing more harm than more conservative treatment. The term "happy hypoxic" was introduced to label patients who had no increased work of breathing though their oxygen saturations were in the mid-80s on supplemental oxygen.

Though the ARDS Berlin criteria based on $\mathrm{PaO} 2 / \mathrm{Fio} 2$ ratio was used to classify mild, moderate and severe cases, treatment of the same was slightly different when it came to COVID-positive patients, relative to other ARDS patients. ${ }^{12}$ Most of the mild COVID ARDS and some moderate to severe COVID ARDS patients did exceptionally well on supplemental oxygen (nasal cannula) or advanced therapies like high flow nasal cannula/noninvasive positive pressure ventilation, avoiding intubation and ventilation. The key to managing such patients was close observation for any respiratory deterioration and immediate intubation by an expert airway team. However, if a patient on admission has increased work of breathing (respiratory distress, increased respiratory rate) and is requiring high volumes of oxygen to maintain acceptable oxygen saturation (severe ARDS), it is prudent to intubate such patients right away. Additionally, in patients with severe ARDS/refractory hypoxemia, we used prone ventilation and neuromuscular blockers in cases of ventilator dyssynchrony.

At our institution, a designated "Tiger Team," operated by the Department of Anesthesiology, responds to and provides airway management for all airway emergencies, COVID and non-COVID. In COVID patients, this team also places invasive hemodynamic monitoring and central lines to limit the exposure of hospital personnel and more quickly and efficiently secure these lines. This team is equipped with full PPE, including powered air-purifying respirators (PAPRs), video laryngoscopy and other advanced airway tools and point-of-care ultrasound.

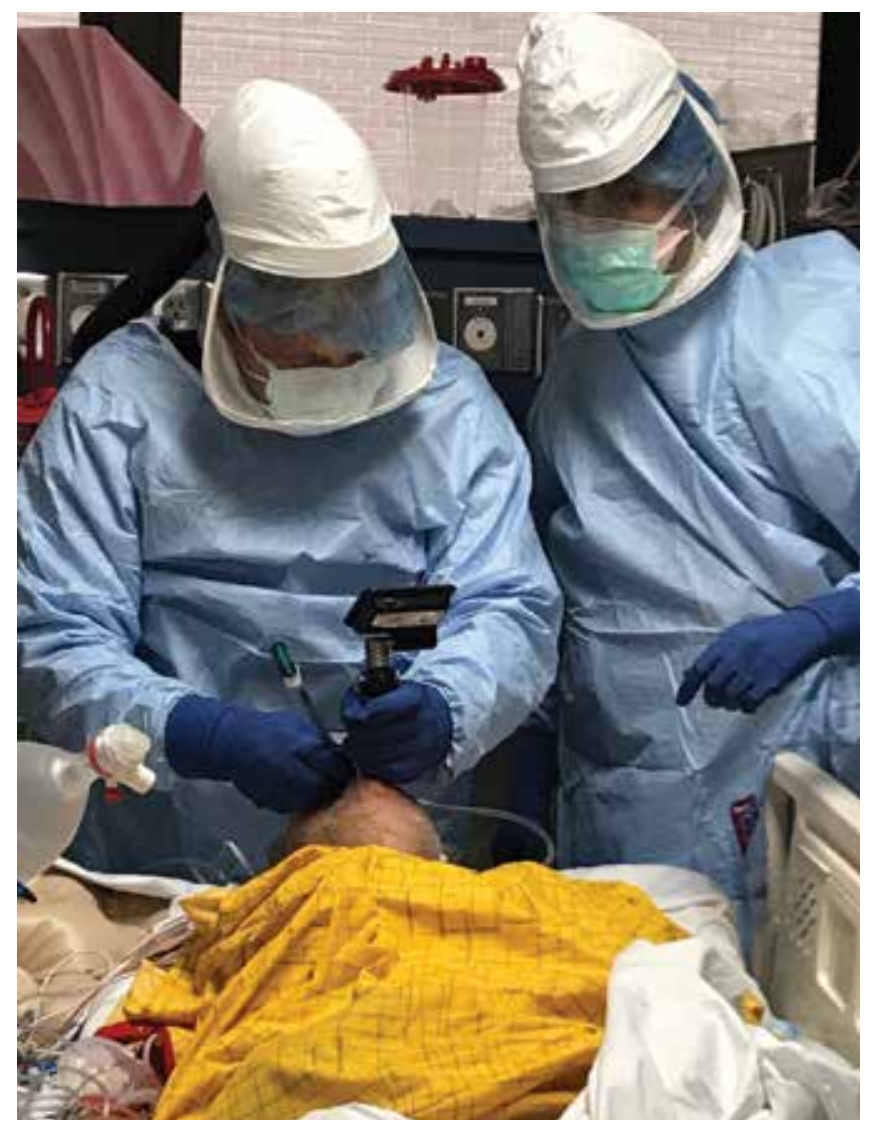

The latest NIH guidelines regarding ventilatory support for COVIDpositive patients are as follows: ${ }^{13}$

- For adults with COVID-19 who are receiving supplemental oxygen, the COVID-19 NIH Guideline Panel recommends close monitoring for worsening respiratory status and in the event intubation becomes necessary, that an experienced practitioner performs the procedure in a controlled setting.

- For adults with COVID-19 and acute hypoxemic respiratory failure despite conventional oxygen therapy, the NIH panel recommends high-flow nasal cannula (HFNC) oxygen over noninvasive positive pressure ventilation (NIPPV).

- In the absence of an indication for endotracheal intubation, the $\mathrm{NIH}$ panel recommends a closely monitored trial of NIPPV for adults with COVID-19 and acute hypoxemic respiratory failure for whom HFNC is not available.

- For mechanically ventilated adults with COVID-19 and acute respiratory distress syndrome (ARDS), the NIH panel recommends using low tidal volume (Vt) ventilation (Vt 4-8 mL/ $\mathrm{kg}$ of predicted body weight) over higher tidal volumes ( $\mathrm{Vt}>8 \mathrm{~mL} /$ $\mathrm{kg})$.

- For mechanically ventilated adults with COVID-19 and refractory hypoxemia despite optimized ventilation, the NIH panel recommends prone ventilation for 12-16 hours per day. 
- For mechanically ventilated adults with COVID-19, severe ARDS and hypoxemia despite optimized ventilation and other rescue strategies, the NIH panel recommends a trial of inhaled pulmonary vasodilator as a rescue therapy. If no rapid improvement in oxygenation is observed, the patient should be tapered off treatment.

- The NIH panel recommends using, as needed, intermittent boluses of neuromuscular blocking agents (NMBA), or continuous NMBA infusion, to facilitate protective lung ventilation.

- In the event of persistent ventilator dyssynchrony that places the patient at risk for ventilator-induced lung injury, the need for ongoing deep sedation, prone ventilation or persistently high plateau pressures, the panel recommends using a continuous NMBA infusion for up to 48 hours as long as patient anxiety and pain can be adequately monitored and controlled.

- There is insufficient data to recommend either for or against the routine use of extracorporeal membrane oxygenation for patients with COVID-19 and refractory hypoxemia. (This has been used at our institution, with limited success). Table 1 shows the labs we order at our institution, depending on disease severity.

\section{TABLE 1:}

Labs ordered depending on disease severity.

\begin{tabular}{|c|c|c|c|}
\hline TIMING & MILD & MODERATE & SEVERE \\
\hline & $\begin{array}{l}\text { Patient with mild } \\
\text { clinical symptoms } \\
\text { RR<24 breaths } \\
\text { per minute } \\
\text { SpO2 }>94 \% \text { on } \\
\text { room air }\end{array}$ & $\begin{array}{l}\text { Symptomatic } \\
\text { patient with mild } \\
\text { to moderate } \\
\text { pneumonia } \\
\text { RR } 24-30 \\
\text { breaths per } \\
\text { minute } \\
\text { SpO2>94\% on } \\
\text { room air }\end{array}$ & $\begin{array}{l}\begin{array}{l}\text { Symptomatic } \\
\text { patient in ARDS } \\
\text { and septic shock }\end{array} \\
\text { RR }>30 \text { breaths } \\
\text { per minute } \\
\text { SpO2 }<94 \% \text { on } \\
\text { room air } \\
\begin{array}{l}\mathrm{PaO} 2 / \mathrm{FiO} 2<300 \\
\text { mmHg or lung } \\
\text { infiltrates }>50 \%\end{array}\end{array}$ \\
\hline $\begin{array}{l}\text { At } \\
\text { admission }\end{array}$ & $\begin{array}{l}\text { CBC, BMP, ECG } \\
\text { HbA1C9 (if } \\
\text { diabetic) } \\
\text { D-dimer }\end{array}$ & $\begin{array}{l}\text { CBC, BMP, ECG } \\
\text { D-dimer, } \\
\text { CRP, serum } \\
\text { ferritin, LDH, } \\
\text { procalcitonin, } \\
\text { troponin, PT/ } \\
\text { INR, ABG, blood } \\
\text { culture (if WBC } \\
\text { count is high), IL- } \\
\text { 6, serum cortisol, } \\
\text { CXR, CT thorax, } \\
\text { cardiac echo }\end{array}$ & $\begin{array}{l}\text { CBC, BMP, ECG } \\
\text { D-dimer, } \\
\text { CRP, serum } \\
\text { ferritin, LDH, } \\
\text { procalcitonin, } \\
\text { troponin, PT/ } \\
\text { INR, ABG, blood } \\
\text { culture (if WBC } \\
\text { count is high), IL- } \\
\text { 6, serum cortisol, } \\
\text { CXR, CT thorax, } \\
\text { cardiac echo, NT } \\
\text { proBNP, serum } \\
\text { magnesium, } \\
\text { serum calcium }\end{array}$ \\
\hline $\begin{array}{l}\text { Repeat } \\
\text { daily }\end{array}$ & & CBC, BMP, ABG & $\mathrm{CBC}, \mathrm{BMP}, \mathrm{ABG}$ \\
\hline $\begin{array}{l}\text { Repeat } \\
\text { every } 72 \\
\text { hrs }\end{array}$ & $\begin{array}{l}\text { D-dimer (if initial } \\
\text { is high) }\end{array}$ & $\begin{array}{l}\text { CRP, D-dimer, } \\
\text { serum ferritin, } \\
\text { LDH, CXR }\end{array}$ & $\begin{array}{l}\text { CRP, D-dimer, } \\
\text { serum ferritin, } \\
\text { LDH, CXR }\end{array}$ \\
\hline
\end{tabular}

\section{PHARMACOLOGIC INTERVENTIONS}

\section{Antivirals/immunomodulators}

All our ICU patients received hydroxychloroquine initially. However, after multiple trials showed no beneficial effects using hydroxychloroquine for either prophylaxis or treatment of COVID patients, we stopped using the drug at our institution. Intensivists at our hospital and other centers have used interleukin- 6 inhibitors and convalescent plasma for severely critically ill patients.

After promising trial results and emergency use authorization by the FDA for remdesivir, we are using it at our institution for treatment of suspected or laboratory-confirmed COVID-19 in adults and children hospitalized with severe disease. Severe disease is defined as patients with oxygen saturation $(\mathrm{SpO} 2) \leq 94 \%$ on room air or requiring supplemental oxygen, invasive mechanical ventilation or extracorporeal membrane oxygenation (ECMO).

For adults, we are using a single loading dose of $200 \mathrm{mg}$ infused intravenously over 30-120 minutes on day 1 , followed by once-daily maintenance doses of $100 \mathrm{mg}$ infused intravenously over 30-120 minutes for four days (days two through five). A treatment course of 10 days is recommended for adults and pediatric patients requiring invasive mechanical ventilation and/or ECMO. A treatment course of five days is recommended for adults and pediatric patients not requiring invasive mechanical ventilation and/or ECMO. If a patient does not demonstrate clinical improvement, treatment may be extended for up to five additional days (i.e., up to a total of 10 days).

According to the NIH Panel ${ }^{13}$ preliminary data from a multinational, randomized, placebo-controlled trial (Adaptive COVID-19 Treatment Trial [ACTT]) of hospitalized patients with COVID-19 showed that patients who were randomized to receive remdesivir had a shorter time to clinical recovery than those who received the placebo. There is not enough clinical trial data to assess the role of remdesivir for patients with mild to moderate COVID-19.

The NIH panel recommends the investigational antiviral agent remdesivir for the treatment of COVID-19 in hospitalized patients with severe disease (defined as having SpO2 $\leq 94 \%$ on ambient air [at sea level], requiring supplemental oxygen, mechanical ventilation or ECMO. Remdesivir is not approved by the FDA; however, it is available through an FDA emergency use authorization (EUA) for the treatment of hospitalized adults and children with COVID-19 and is currently being investigated in clinical trials. Remdesivir is also available through an emergency access program for children ( $<18$ years of age) and pregnant patients. Additional data on the use of remdesivir for patients with COVID-19, including analyses of important patient subgroups, is pending and might change the recommendations. The NIH panel does not recommend remdesivir for the treatment of mild or moderate COVID-19 outside of a clinical trial. 


\section{Drugs Not Recommended by the Panel}

Except in the context of a clinical trial, the panel recommends against the use of the following drugs for the treatment of COVID-19:

- High-dose chloroquine (600 mg twice daily for 10 days) for the treatment of COVID-19

- The combination of hydroxychloroquine plus azithromycin because of the potential for toxicities and prolonged QT interval

- Lopinavir/ritonavir or other HIV protease inhibitors because of unfavorable pharmacodynamics and negative clinical trial data

Insufficient Data to Recommend Either for or Against the Use:

- Though most of the hospitals initially used hydroxychloroquine, the NIH highlights there is insufficient clinical data to recommend either for or against using chloroquine or hydroxychloroquine for the treatment of COVID-19. As new data is published, the consensus seems to be that there is no clear benefit to its use and most institutions are abandoning the therapy, as is the case at our institution.

- Interleukin-1 inhibitors (e.g., anakinra)

-Interleukin-6 inhibitors (e.g., sarilumab, siltuximab, tocilizumab)

- Also, the NIH panel states that there is insufficient data to recommend either for or against the use of COVID-19 convalescent plasma or SARS-CoV-2 immune globulins for the treatment of COVID-19.

\section{Antibiotics}

At our institution, some intensivists treating patients with COVID-19 routinely administer broad-spectrum antibiotics to all patients with moderate or severe hypoxemia. Other intensivists administered antibiotics only for specific situations, such as the presence of a lobar infiltrate on chest x-ray, leukocytosis, elevated serum lactate, microbiologic data or shock. Per the latest NIH guidelines, in patients with COVID-19 and severe or critical illness, there is insufficient data to recommend empiric broad-spectrum antimicrobial therapy in the absence of another indication. ${ }^{13}$

\section{Corticosteroids}

Initially, at our institution, we used low dose corticosteroids only in severely critically ill COVID-positive patients with refractory shock or myocarditis. However, after the data from the RECOVERY trial done in the U.K. came out last month, we are now using dexamethasone for COVID-19 patients. Our institution's recommendation is as follows:

Recommend consideration of dexamethasone in COVID-19 patients who are hypoxemic, defined as:

- SpO2 $\leq 94 \%$ on ambient air at rest

- Requiring supplemental $\mathrm{O} 2$

- Mechanically ventilated or on ECMO
Our institution does not recommend dexamethasone in COVID-19 patients who do not have hypoxemia due to an increased risk of harm/potential mortality in this subset of patients noted in the RECOVERY trial.

Our institution does not recommend dexamethasone in ambulatory/outpatient COVID-19 patients at this time.

\section{Dosing and Duration of Therapy}

Dexamethasone dosing is $6 \mathrm{mg}$ PO (or IV) daily for up to 10 days OR until the patient is discharged from the hospital, whichever is sooner. The $\mathrm{NIH}$ panel recommends using dexamethasone (at a dose of $6 \mathrm{mg}$ per day for up to 10 days) for the treatment of COVID-19 in patients who are mechanically ventilated (AI) and in patients who require supplemental oxygen but who are not mechanically ventilated $(\mathrm{BI})$. The $\mathrm{NIH}$ panel recommends against using dexamethasone for the treatment of COVID-19 in patients who do not require supplemental oxygen. $(\mathrm{Al})^{13,14}$

\section{Antithrombotic Therapy}

The decision of whether and when to start therapeutic anticoagulation in COVID-positive patients with abnormal coagulation parameters (D-dimer, fibrinogen) has been a very controversial topic among our intensivists. Many hospitals have their criteria to start anticoagulation. At our institution, we started therapeutic anticoagulation only if we suspected venous or arterial thrombosis in a patient.

From the NIH guideline, hospitalized adults with COVID-19 should receive VTE prophylaxis per the standard of care for other hospitalized adults. It also recommends patients with COVID-19, who experience a thromboembolic event or who are highly suspected of having the thromboembolic disease (when imaging is not possible), be managed with therapeutic doses of anticoagulant therapy as per the standard of care for patients without COVID-19. The NIH further states that there is currently insufficient data to recommend for or against routine deep vein thrombosis screening in COVID-19 patients without signs or symptoms of VTE, regardless of the status of their coagulation markers. Lastly, NIH recommends against routinely discharging COVID patients on VTE prophylaxis. ${ }^{13}$

\section{CONCOMITANT MEDICATIONS}

\section{Angiotensin-Converting Enzyme (ACE) Inhibitors and Angiotensin Receptor Blockers (ARBs)}

According to various studies, angiotensin-converting enzyme 2 (ACE2) is the cell surface receptor in severe acute respiratory syndrome coronavirus 2 (SARS-CoV-2). ${ }^{15}$ It has been hypothesized that the modulation of ACE2 associated with these therapies could suppress or enhance SARS-CoV-2 replication. Investigations of the role of ARBs and recombinant human ACE2 in treatment and prevention of SARS-CoV-2 infection are underway. It is unclear whether these medications are helpful, harmful or neutral in the pathogenesis of SARS-CoV-2 infection. Currently, there is a lack of sufficient clinical evidence demonstrating that ACE inhibitors or ARBs have any impact on the susceptibility of individuals to SARSCoV-2 or on the severity or outcomes of infection. At our institution, we continued ACEIs/ARBs if patients were on them previously but 
did not start it as a new medication.

The NIH panel recommends against the use of ACE inhibitors or ARBs for the treatment of COVID-19 outside of the setting of a clinical trial.

The panel also recommends persons with COVID-19 who are prescribed ACE inhibitors or ARBs for cardiovascular disease (or other indications) continue these medications. ${ }^{13}$

\section{HMG-CoA Reductase Inhibitors (Statins)}

HMG-CoA reductase inhibitors, or statins, affect ACE2 as part of their function in reducing endothelial dysfunction. It has been proposed that these agents have a potential role in managing patients with severe COVID-19. Observational studies have reported that statin therapy may reduce cardiovascular morbidity in patients admitted with other respiratory infections, such as influenza and bacterial pneumonia.

At our institution, we did not routinely start statins on our ICU patients. The $\mathrm{NIH}$ panel recommends against the use of statins for the treatment of COVID-19 outside of the setting of a clinical trial. The NIH panel also recommends that persons with COVID-19, who are prescribed statin therapy for the treatment or prevention of cardiovascular disease, continue these medications. ${ }^{13}$

\section{Nonsteroidal Anti-Inflammatory Drugs (NSAIDs)}

It has been proposed that NSAIDs, like ibuprofen, can increase the expression of ACE2 and inhibit antibody production. Shortly after these reports, the FDA stated that there is no evidence linking the use of NSAIDs with worsening of COVID-19 and advised patients to use NSAIDs as directed. At our institution, we continued NSAIDS if the patient was taking it previously and alternated them with acetaminophen as antipyretics. Per NIH guidelines, persons with COVID-19 who are taking NSAIDs for a comorbid condition should continue therapy as previously directed by their physician. The panel also recommends that there be no difference in the use of antipyretic strategies (i.e., with acetaminophen or NSAIDs) in patients with or without COVID-19.

\section{POST COVID COMPLICATIONS AND FOLLOW-UP}

There have been reports of patients suffering sequelae after mild/ moderate and severe COVID infection. This may place an additional burden on primary health care professionals to monitor and treat such patients in a so-called "post COVID" clinic. Additionally, these patients may require long-term physical therapy and rehabilitation.

A post-viral syndrome known to occur with other coronaviruses, like SARS and MERS, called chronic fatigue syndrome/myalgic encephalomyelitis (CFS/ME), has also been occurring in COVID patients after recovery. These patients present with symptoms such as persistent fatigue, brain fog, diffuse myalgia, depression and non-restorative sleep. Patients with underlying comorbidities like obesity, hypertension and diabetes are more prone to this syndrome.

A possible etiology of the syndrome is the disturbance of the lymphatic drainage from the microglia in the brain. The main pathways of the lymphatic drainage of the brain are via the perivascular spaces along the olfactory nerves through the cribriform plate into the nasal mucosa. The symptom of anosmia seen in some COVID patients might be explained if the pathogenesis affects a similar pathway. ${ }^{16,17}$ Some patients are also presenting with dysautonomia features, like chest pain, rapid heartbeat, anxiety, numbness, dizziness, low blood pressure, shortness of breath and gastrointestinal problems that are caused by an imbalance in the autonomic nervous system. It has been reported that more young patients present with dysautonomia symptoms post-recovery who have had only mild to moderate COVID symptoms during the acute phase of the disease. ${ }^{16,17} \mathrm{~A}$ few patients are presenting with Guillain-Bare syndrome 10-15 days after initial COVID onset. There is a report of one patient developing the syndrome approximately three months after initial COVID symptoms. ${ }^{18}$

A subsection of COVID patients are having strokes and encephalitis in the acute phase and will eventually have to deal with sequelae from these above clinical conditions.

Some of the COVID patients with a severe presentation during hospitalization have had myocarditis and decreased cardiac function that may continue during recovery. Patients with previous cardiac conditions should be followed closely by their primary care physician.

Since COVID is a respiratory virus that primarily targets the lungs, there have been studies and reports indicating reduced lung capacity in the post-recovery period. Lung scarring leading to pulmonary fibrosis has also been reported. Even young patients have reported reduced exercise tolerance in the post-recovery period. 17

Finally, the common problems of a prolonged ICU stay, such as critical care neuropathy and myopathy may be encountered postdischarge. It is imperative to follow such patients in an outpatient clinic to monitor and provide appropriate care. The COVID-19 virus is not stimulating a robust antibody response in patients with mild to moderate symptoms and even with severe disease, it has been reported that antibodies may only last for a few months. Thus, reinfection is a real possibility and the primary care physician should be aware of re-infection chances in recovered COVID patients. ${ }^{19}$

\section{CONCLUSION}

As COVID-19 is a novel disease, we are learning new information every day with regards to pathophysiology, symptomatology, immunity and treatment options. It is essential to be aware of the latest NIH/CDC recommendations regarding COVID-19 and keep ourselves updated. Doing so may help us to achieve greater success and save many more lives during a potential second and subsequent waves of the pandemic. 


\section{AUTHOR DISCLOSURE(S):}

The author(s) declare no relevant financial affiliations or conflicts of interest.

\section{REFERENCES:}

1. Coronavirus Disease (COVID-19) Situation Reports. World Health Organization. https://www.who.int/emergencies/diseases/novelcoronavirus-2019/situation-reports/. Published 2020. Accessed August $3,2020$.

2. Coronavirus disease 2019 (COVID-19): cases in U.S. 2020. Centers for Disease Control and Prevention. https://www.cdc.gov/coronavirus/2019ncov/cases-updates/cases-inus.html. Published July 11, 2020. Accessed August 3, 2020.

3. Wu C, Chen X, Cai Y, et al. Risk Factors Associated with Acute Respiratory Distress Syndrome and Death in Patients with Coronavirus Disease 2019 Pneumonia in Wuhan, China [published online ahead of print, 2020 Mar 13]. JAMA Intern Med. 2020;180(7):1-11. doi:10.1001/ jamainternmed.2020.0994

4. Guan W-J, Ni Z-Y, Hu Y, et al. Clinical Characteristics of Coronavirus Disease 2019 in China. New England Journal of Medicine. 2020;382(18):1708-1720.doi:10.1056/nejmoa2002032

5. Qingxian C, Fengjuan C, Fang L, et al. Obesity and COVID-19 Severity in a Designated Hospital in Shenzhen, China. Preprints with the Lancet. 2020. doi:10.2139/ssrn.3556658

6. Centers for Disease Control and Prevention. Coronavirus disease 2019 (COVID-19): People who are at higher risk for severe illness. 2020. https:// www.cdc.gov/coronavirus/2019-ncov/need-extra-precautions/people-athigher-risk.html. Accessed April 8, 2020.

7. Garg S, Kim L, Whitaker M, et al. Hospitalization Rates and Characteristics of Patients Hospitalized with Laboratory-Confirmed Coronavirus Disease 2019 - COVID-NET, 14 States, March 1-30, 2020. MMWR Morb Mortal Wkly Rep. 2020;69(15):458-464. Published 2020 Apr 17. doi:10.15585/ mmwr.mm6915e3

8. Li Q, Guan X, Wu P, et al. Early Transmission Dynamics in Wuhan, China, of Novel Coronavirus-Infected Pneumonia. N Engl J Med. 2020;382(13):1199-1207. doi:10.1056/NEJMoa2001316

9. Lauer SA, Grantz KH, Bi Q, et al. The Incubation Period of Coronavirus Disease 2019 (COVID-19) From Publicly Reported Confirmed Cases: Estimation and Application. Ann Intern Med. 2020;172(9):577-582. doi:10.7326/M20-0504

10. Wu Z, McGoogan JM. Characteristics of and Important Lessons From the Coronavirus Disease 2019 (COVID-19) Outbreak in China: Summary of a Report of 72314 Cases From the Chinese Center for Disease Control and Prevention [published online ahead of print, 2020 Feb 24]. JAMA. 2020;10.1001/jama.2020.2648. doi:10.1001/jama.2020.2648

11. Wu Z, McGoogan JM. Characteristics of and Important Lessons From the Coronavirus Disease 2019 (COVID-19) Outbreak in China: Summary of a Report of 72314 Cases From the Chinese Center for Disease Control and Prevention [published online ahead of print, $2020 \mathrm{Feb} 24$ ]. JAMA. 2020;10.1001/jama.2020.2648. doi:10.1001/jama.2020.2648

12. Li X, Ma X. Acute respiratory failure in COVID-19: is it "typical" ARDS? Critical Care. 2020;24(1). doi:10.1186/s13054-020-02911-9

13. COVID-19 Treatment Guidelines Panel. Coronavirus Disease 2019 (COVID-19) Treatment Guidelines. National Institutes of Health. Available at https://covid19treatmentguidelines.nih.gov/. Accessed May 15, 2020.

14. Shang L, Zhao J, Hu Y, Du R, Cao B. On the use of corticosteroids for 2019-nCoV pneumonia. Lancet. 2020;395(10225):683-684. doi:10.1016/ S0140-6736(20)30361-5

15. Patel AB, Verma A. COVID-19 and Angiotensin-Converting Enzyme Inhibitors and Angiotensin Receptor Blockers: What Is the Evidence? [published online ahead of print, 2020 Mar 24]. JAMA. 2020;10.1001/ jama.2020.4812. doi:10.1001/jama.2020.4812

16. Perrin R, Riste L, Hann M, Walther A, Mukherjee A, Heald A. Into the looking glass: Post-viral syndrome post COVID-19. Medical Hypotheses. 2020;144:110055.doi:10.1016/j.mehy.2020.110055

17. Carfi A, Bernabei R, Landi F. Persistent Symptoms in Patients After Acute COVID-19. Jama. July 2020. doi:10.1001/jama.2020.12603

18. Toscano G, Palmerini F, Ravaglia S, et al. Guillain-Barré Syndrome Associated with SARS-CoV-2. New England Journal of Medicine. 2020;382(26):2574-2576. doi:10.1056/nejmc2009191

19. Pollán M, Pérez-Gómez B, Pastor-Barriuso R, et al. Prevalence of SARS-CoV-2 in Spain (ENE-COVID): a nationwide, population-based seroepidemiological study. The Lancet. July 2020. doi:10.1016/s01406736(20)31483-5 